\title{
ON THE EXTENSION PROPERTY OF MEASURABLE SPACES
}

\author{
WŁODZIMIERZ BZYL AND ADAM MYSIOR
}

\begin{abstract}
We prove that a metrizable measurable space has the extension property if and only if it is isomorphic to a Borel subset of the real line. It follows, in particular, that $(\mathbf{R}, \mathcal{P}(\mathbf{R}))$ does not have the extension property. Both results answer the questions raised by $R$. M. Shortt.
\end{abstract}

1. Introduction. A measurable space is a pair $(X, X)$, where $X$ is a nonempty set and $X$ is a $\sigma$-field of subsets of $X$. A measurable space $(A, A)$ is a subspace of a measurable space $(X, X)$ provided that $A \subseteq X$ and $A=\{A \cap B: B \in X\}$. For measurable spaces $(X, X)$ and $(Y, Y)$ a function $f: X \rightarrow Y$ is measurable if $f^{-1}(C) \in \mathcal{X}$ for any $C \in \mathcal{Y}$.

Recently, R. M. Shortt introduced in $[\mathbf{S}]$ the notion of the extension property of measurable spaces. Namely, a measurable space $(Y, \mathcal{Y})$ is said to have the extension property if for every measurable space $(X, \mathcal{X})$ and every subspace $(A, \mathcal{A})$ of $(X, \mathcal{X})$, each measurable function $f: A \rightarrow Y$ may be extended to a measurable function $\tilde{f}: X \rightarrow Y$.

The purpose of this paper is to reduce the possible number of spaces which have the extension property. We prove the following

THEOREM 1. Let $(Y, Y)$ be a measurable space. If there exists a metric on $Y$ such that the associated Borel $\sigma$-field is a proper subfamily of $\mathcal{Y}$, then $(Y, \mathcal{Y})$ does not have the extension property.

This theorem yields, in particular, the following corollary which answers a question raised in $[\mathbf{S}]$.

COROLlARY. $(\mathbf{R}, \mathcal{P}(\mathbf{R}))$ does not have the extension property.

A measurable space $(Y, Y)$ is called separated if for every two distinct points of $Y$ there exists a set in $\mathcal{Y}$ which contains one of them but not the other. R. M. Shortt proved in $[\mathbf{S}]$ that a countably generated and separated measurable space has the extension property if and only if it is a standard space, i.e. it is isomorphic to a Borel subset of the real line.

Let a measurable space $(Y, Y)$ be metrizable (submetrizable) provided that there exists a metric on $Y$ such that $\mathcal{Y}$ is (resp. contains) the associated $\sigma$-field of Borel sets. Observe that

countable generated and separated $\Rightarrow$ metrizable $\Rightarrow$ submetrizable $\Rightarrow$ separated.

Received by the editors November 29, 1983.

1980 Mathematics Subject Classification. Primary 28A20; Secondary 03E15, 03 E55.

Key words and phrases. Measurable space, extension property, standard space, metrizable measurable space, analytic set.

(C) 1984 American Mathematical Society $0002-9939 / 84 \$ 1.00+\$ .25$ per page 
R. M. Shortt raised in $[\mathbf{S}]$ the problem how to decide which spaces among metrizable ones do have the extension property. We settle this for the wider class of submetrizable spaces by proving the following

THEOREM 2. Among submetrizable measurable spaces those having the extension property are precisely the standard spaces.

COROLlARY. Among metrizable measurable spaces those having the extension property are precisely the standard spaces.

The analogous reduction within the class of all separated measurable spaces does not hold. We conclude the paper with an example of a $\sigma$-field $\mathcal{Y}$ on $\mathbf{R}$ such that $(\mathbf{R}, \mathcal{Y})$ has the extension property, $\mathcal{Y}$ contains singletons and yet $(\mathbf{R}, \mathcal{Y})$ is not a standard space.

2. Key lemmas. Our theorems are based on the following two lemmas.

LEMMA 1. If a measurable space $(Y, \mathcal{Y})$ has the extension property, then for every Polish space $Z$ and for every measurable function $g: Y \rightarrow Z$ the image $g(Y)$ is an analytic subset of $Z$.

ProOF. Assume that a measurable space $(Y, Y)$ has the extension property. Let $Z$ be an arbitrary Polish space and $g: Y \rightarrow Z$ be an arbitrary measurable function. We prove that $g(Y)$ is an analytic subset of $Z$.

For every $A \in \mathcal{Y}$ denote by $S_{A}$ the subset of $2^{\mathcal{P}(Y)}$ consisting of the all function $s: \mathcal{P}(Y) \rightarrow\{0,1\}$ such that $s(A)=1$. Consider a measurable space $\left(2^{\mathcal{P}(Y)}, B_{y}\right)$, where $B y$ is a $\sigma$-field generated by $\left\{S_{A}: A \in \mathcal{Y}\right\}$.

Observe at first that there exists a measurable function $j$ from $2^{\mathcal{P}(Y)}$ onto $Y$. Indeed the generalized Marczewski function $i: Y \rightarrow 2^{\mathcal{P}(Y)}$, defined by $i(y)(A)=1$ if $y \in A$ and $i(y)(A)=0$ if $y \notin A$, is a measurable isomorphism of $Y$ and $i(Y)$. Since the measurable space $(Y, Y)$ has the extension property, the measurable function $i^{-1}$ from $i(Y)$ onto $Y$ has some measurable extension $j$ from $2^{\mathcal{P}(Y)}$ onto $Y$.

Consider now the measurable function $g \circ j: 2^{\mathcal{P}(Y)} \rightarrow Z$. Observe that $g(Y)=$ $g \circ j\left(2^{\mathcal{P}(Y)}\right)$. Since the $\sigma$-field of all Borel subsets of $Z$ is countably generated, the function $g \circ j$ is measurable with respect to some countably generated sub- $\sigma$-field of $B y$. It follows that there exists a countable family $C \subseteq y$ such that $g \circ j$ is measurable with respect to the $\sigma$-field generated by $\left\{S_{A}: A \in C\right\}$. The subspace $C=\left\{s \in 2^{\mathcal{P}(Y)}: s(A)=0\right.$ for $\left.A \notin C\right\}$ of $2^{\mathcal{P}(Y)}$ is isomorphic with the Cantor set. Since $g \circ j$ depends only on coordinates indexed by the members of $C$, we have $g \circ j\left(2^{\mathcal{P}(Y)}\right)=g \circ j(C)$ and therefore $g(Y)$ is an analytic subset of $Z$.

A subfamily $\mathcal{F}$ of a family of $\mathcal{Y}$ is called $\mathcal{Y}$-additive provided that $\bigcup \mathcal{F}_{0} \in \mathcal{Y}$ for every $\mathcal{F}_{0} \subseteq \mathcal{F}$.

LEMMA 2. If a measurable space $(Y, \mathcal{Y})$ has the extension property then every disjoint $\mathcal{Y}$-additive subfamily of $\mathcal{Y}$ is countable.

PROOF. Assume on the contrary that there exists a disjoint uncountable $y$ additive subfamily $₹$ of $\mathcal{Y}$. Clearly, we may assume that $\mathcal{F}$ covers $Y$ and has cardinality $\aleph_{1}$. Choose on the real line a nonanalytic subset $T$ with cardinality $\aleph_{1}$. Since the partition $\mathcal{F}$ is $\mathcal{Y}$-additive, the function $g: Y \rightarrow R$ which sends in one-to-one manner all members of $₹$ onto the points of $T$ is measurable. On the other hand $g(Y)=T$, which contradicts Lemma 1 . 
REMARK. If a measurable space $(Y, \mathcal{Y})$ has the extension property, then every disjoint subfamily of $\mathcal{Y}$ has cardinality $\leq 2^{\aleph_{0}}$. Indeed, by the proof of Lemma 1 there exists a measurable function $j$ from $\left(2^{\mathcal{P}(Y)}, B_{y}\right)$ onto $(Y, Y)$. It suffices therefore to observe that every disjoint subfamily of $B_{y}$ has cardinality $\leq 2^{\aleph_{0}}$. This follows however from the fact that each member of $B y$ depends on countably many coordinates and from the Erdös-Rado intersection theorem (cf. [D] with $a=$ $2, b=\aleph_{0}$ or $[\mathbf{J}$, Appendix 2]).

\section{Proofs of the theorems.}

ProOF OF THEOREM 1. Assume that $(Y, \mathcal{Y})$ has the extension property. Let $B \subseteq Y$ be a $\sigma$-field of Borel sets with respect to some metric $d$ on $Y$. We prove that $B=Y$.

Observe that the metric space $(Y, d)$ has to be separable. Otherwise, it would contain an uncountable discrete closed subset which implies that $\mathcal{Y}$ would have an uncountable $\mathcal{Y}$-additive subfamily of singletons and this contradicts Lemma 2 . We may therefore assume that the measurable space $(Y, B)$ is a subspace of some Polish space $(Z, Z)$. To prove that $B=\mathcal{Y}$ it suffices to show that $A \in B$ for every $A \in \mathcal{Y}$ with $\phi \neq A \neq Y$. Choose a point $y_{0} \in A$ and define the function $g: Y \rightarrow Z$ by letting $g(y)=y$ if $y \in A$ and $g(y)=y_{0}$ if $y \in Y \backslash A$. It is easy to check that $g$ is a measurable function from $(Y, \mathcal{Y})$ to $(Z, Z)$ and $g(Y)=A$. Hence, following Lemma $1, A$ is an analytic subset of $Z$. By the same argument, $Y \backslash A$ is an analytic subset of $Z$. By the Luzin Separation Theorem [K, $\S 39$.III] there exists a Borel subset $B$ of $Z$ such that $A \subseteq B$ and $B \cap(Y \backslash A)=\emptyset$. Hence $A=B \cap Y \in B$ which ends the proof.

PROOF OF THEOREM 2. Let $(Y, \mathcal{Y})$ be a submetrizable measurable space which has the extension property. We prove that $(Y, \mathcal{Y})$ is a standard space.

By the assumption there exists a metric $d$ on $Y$ such that $B \subseteq \mathcal{Y}$, where $B$ is a $\sigma$ field of all Borel sets with respect to $d$. Theorem 1 implies that $B=\mathcal{Y}$. Analogously as in the previous proof the metric space $(Y, d)$ has to be separable. Hence $y$ is countably generated and by Shortt's theorem mentioned in the Introduction, $(Y, \mathcal{Y})$ is a standard space.

4. Example. Let $B$ be the $\sigma$-field of all Borel subsets of $[0, \infty)$. For every $B \in B$ put $-B=\{-r: r \in B\}$ and consider the $\sigma$-field $\mathcal{Y}$ of subsets of $\mathbf{R}$ generated by the family $\{r: r \in \mathbf{R}\} \cup\{-B \cup B: B \in B\}$. We show that $(\mathbf{R}, \mathcal{Y})$ has the extension property. Observe that $\mathcal{Y}$ contains points but is not countably generated. Hence $(\mathbf{R}, \mathcal{Y})$ is separated but is not a standard space.

Let $(A, \mathcal{A})$ be a subspace of some measurable space $(X, X)$ and let $f$ be a measurable function from $(A, \mathcal{A})$ to $(\mathbf{R}, \mathcal{Y})$. We are to find a measurable extension $\tilde{f}$ of $f$ onto $(X, X)$. Observe that the absolute value $|f|$ is a measurable function from $(A, A)$ to $([0, \infty), B)$. Since $([0, \infty), B)$ is a standard space, the function $|f|$ has some measurable extension $\widetilde{|f|}$ onto $(X, \mathcal{X})$. For every $r \in[0, \infty)$ pick a set $E_{r} \in \mathcal{X}$ such that $f^{-1}(r)=A \cap E_{r}$. Now define $\tilde{f}: X \rightarrow \mathbf{R}$ by letting $\tilde{f}(x)=r$ if $\widetilde{\mid f} \mid(x)=r$ and $x \in E_{r}$, and $\tilde{f}(x)=-r$ if $\widetilde{|f|} \mid(x)=r$ and $x \notin E_{r}$. It is easy to see that such an $\tilde{f}$ extends $f$. To show that $\tilde{f}$ is a measurable function from $(X, \mathcal{X})$ to $(\mathbf{R}, \mathcal{Y})$ it suffices to check that $\tilde{f}^{-1}(r)$ equals $\left.\widetilde{|f|}\right|^{-1}(r) \cap E_{r},\left.\widetilde{|f|}\right|^{-1}(|r|) \backslash E_{r}$ or $\left.\widetilde{|f|}\right|^{-1}(0)$ depending on whether $r<0, r>0$, or $r=0$ and that $\tilde{f}^{-1}(-B \cup B)=\widetilde{\mid f}^{-1}(B)$. 


\section{REFERENCES}

[D] R. O. Davies, An intersection theorem of Erdös and Rado, Proc. Cambridge Philos. Soc. 63 (1967), 995-996.

[J] I. Juhasz, Cardinal functions in topology, Math. Centre Tracts, Vol. 34, Math. Centrum, Amsterdam, 1971.

[K] K. Kuratowski, Topology, Vol. I, Academic Press, New York and London; PWN, Warsaw, 1966.

[S] R. M. Shortt, The extension of measurable functions, Proc. Amer. Math. Soc. 87 (1983), 444-446.

Instytut MATEMATyki, Uniwersytet GDAŃSki, Wita Stwosza 57, 80-952 GDAŃSK, POLAND 